chemical activity. This result is in accordance with expectations.

(2) Constantinides has already found that the current which passes between two electrodes of different areas bathed in the afterglow is proportional to the area of the cathode, and has therefore concluded that the conductivity of active nitrogen is due to emission of electrons by the metal, either photoelectrically or else under bombardment by the luminous gas. We find that if the electrodes are placed in a thin quartz vessel surrounded by, but not actually containing, glowing nitrogen, no current passes, even under the most varied conditions of experiment both in the electrode chamber and outside. We therefore conclude either that the effect is produced by light of a wave-length less than $1400 \mathrm{~A}$., or that the second of Constantinides' theories is correct, and that it may be regarded as evidence for the presence of a metastable form of nitrogen which is deactivated by the metal surfaces, with emission of electrons, as in the experiments of Oliphant upon metastable atoms of helium. When the electrodes are immersed in the glowing gas, the current depends upon the area of the cathode, as found by Constantinides, but also upon the metal of which it is composed. Attempts are being made to correlate it with the workfunction of the metal ; such estimates as we have been able to obtain suggest something greater than 4 volts as the minimum energy of the metastable body causing the emission of electrons.

(3) When the glow is destroyed by heating the gas before it reaches the electrodes, the conductivity also vanishes, but the concentration of chemically active nitrogen remains unchanged, as already found by one of us (E. J. B. W.); the observation seems to indicate that another modification of nitrogen is present which is chemically inactive, but able to cause metals to emit electrons, and possesses an energy of not less than about 4 volts. It may be noted that the CarioKaplan theory provides for the existence of metastable molecules of c. 8 volts, the destruction of the glow by heat being due on the same theory to the deactivation of these molecules on hot surfaces.

(4) We have been able to correlate the observed conductivity and glow intensity with the nature and concentration of the photogens, or other gases, present in active nitrogen.

E. J. B. WILLEY.

W. A. Stringfellow.

Chemistry Department,

University College, W.C.1, July 23.

\section{Raman Displacements and the Infra-red Absorption Bands of Carbon Disulphide.}

THE Raman spectrum of carbon disulphide has been determined by a number of observers (Gavesan and Venkateswaran, Nature, 124, 57 ; 1929 ; Petrikaln and Hochberg, Zeit. phys. Chem., B. 3, 217; 1929 ; and by Schaefer, Matossi, and Anderhold, Phys. Zeit., 30, 584; 1929). In all cases an intense line displaced by $654-658 \mathrm{~cm} .^{-1}$ was observed, together with a weak line corresponding to $795-807 \mathrm{~cm}^{-1}$. The spectrum is of considerable interest, as it has been impossible up to the present to reconcile it with the observed infra-red spectrum as determined by Coblentz.

We have recently reinvestigated the absorption spectrum, using the vapour of carbon disulphide, and have explored the region from $1 \mu$ to $22 \mu$. In this range there are four bands, $A, B, C$, and $D$ at 878 , 1522,2179 , and $2335 \mathrm{~cm}^{-1}$ respectively; of these, owing to its great intensity, $B$ is probably a funda-

mental band, $\nu_{3}$. Now it has been previously assumed (Ghosh and Mahanti, Nature, 124, 230 ; 1929; and Phys. Zeit., 30, 531 ; 1929 ; Snow, Proc. Royal Soc., A 128,311 ; 1930) that the two Raman lines are in effect a doublet of the same nature as is given by carbon dioxide, and that this type of Raman spectrum is characteristic of such linear molecules: on this supposition Ghosh and Mahanti have averaged the frequencies of the two Raman lines and attempted to deduce the infra-red spectrum with this value as a fundamental. Nitrous oxide gives only one line, however, and we believe that the two lines for carbon disulphide have different origins. We postulate two additional fundamental frequencies, $\nu_{2}=655 \mathrm{~cm}$. $^{-1}$ (optically inactive), and $v_{1}=150 \mathrm{~cm} .^{-1}$ approximately. The spectra can now be summarised as in the table below. All frequencies are in wave numbers.

The Absorption Spectrum of Carbon Disulphide VAPOUR IN THE INFRA-RED.

\begin{tabular}{|c|c|c|c|}
\hline Infra-red Band. & Raman Line. & Origin. & Calculated Value. \\
\hline$(150)$ & - & $\nu_{1}$ & - \\
- & 655 & $\nu_{2}$ & - \\
878 & 800 & $\nu_{1}+\nu_{2}$ & 805 \\
1522 & - & $\nu_{3}-\nu_{2}$ & 867 \\
2179 & - & $\nu_{3}$ & - \\
2335 & - & $\nu_{3}+\nu_{2}$ & 2177 \\
\hline
\end{tabular}

Some bands isolated by Coblentz seem to be characteristic of the liquid state as they are absent from our spectrum.

We would add that we have resolved with certainty two of these bands into $P$ and $R$ branches with $a$ frequency difference of $12-13 \mathrm{~cm}^{-1}$. Carbon disulphide, then, is a rectilinear molecule with one moment of inertia, the value of which is approximately $312 \times 10^{-40} \mathrm{gm} . \mathrm{cm}^{2}$

C. R. BALLEY

A. B. D. CASSIE.

Sir William Ramsay Laboratories of Inorganic and Physical Chemistry, University College, London, Aug. 5.

\section{Absorption of Sound at Oblique Incidence.}

In the issue of Nature for July 5 Dr. E. T. Paris discusses some recently published work at the Bureau of Standards on the absorption of sound at oblique angles of incidence. A detailed discussion of Dr. Paris's criticism of our experimental technique does not seem called for at present. These experiments are pioneer work on this subject, and it is much to be desired that others should give the question experimental attention, the more so because the Bureau's results are at variance with previous theoretical conclusions.

Paris (Proc. Roy. Soc., A, 115, p. 407 ; 1927) and Larmor (Proc. Camb. Phil. Soc., 27, part 2, p. 231; April 1930) have investigated the subject mathematically, and have reached conclusions which fail to agree, not only with the Bureau of Standards' results, but also with each other. For grazing incidence, Larmor finds that the absorption should be infinite, while Paris comes to the conclusion that it should be zero.

It would be very desirable that the reasons for this discrepancy in the two theories should be cleared up. It is possible that there is a fundamental inconsistency in the assumption of potential flow and absorption at reflection. This is pointed out in the Bureau of Standards' publication (Bureau of Standards Journal of Research, Feb. 1930). Sound absorption depends

No. 3175, VoL. 126] 